\title{
Factors affecting pupil size after dilatation: the Twin Eye Study
}

\author{
Christopher J Hammond, Harold Snieder, Tim D Spector, Clare E Gilbert
}

\begin{abstract}
Backgroundlaims-Well dilated pupils make eye surgery easier. A classic twin study was established to examine the relative importance of genes and environment in the variance of pupil size after mydriasis, and to examine the effects of other factors such as age, iris colour, and refractive error.

Methods-506 twin pairs, 226 monozygotic (MZ) and 280 dizygotic (DZ), aged 49-79 (mean age 62.2 years, SD 5.7) were examined. Dilated pupil size was measured using a standardised grid superimposed over digital retroillumination images taken 50-70 minutes after mydriasis using tropicamide $1 \%$ and phenylephrine $10 \%$. Univariate maximum likelihood model fitting was used to estimate genetic and environmental variance components.

Results-Dilated pupil size was more highly correlated in $\mathrm{MZ}$ compared with DZ twins (intraclass correlation coefficients 0.82 and 0.39 respectively). A model specifying additive genetic and unique environmental factors showed the best fit to the data, yielding a heritability of 78-80\%. Individual environmental factors explained $18-19 \%$ of the variance in this population. Age only accounted for $2-3 \%$ of the variance and refractive error and iris colour did not significantly contribute to the variance.

Conclusions-Pupil size after mydriasis is largely genetically determined, with a heritability of up to $80 \%$.

(Br f Ophthalmol 2000;84:1173-1176)
\end{abstract}

Twin Research and Genetic Epidemiology Unit, St Thomas's

Hospital, London SE1 7EH, UK

C J Hammond

$\mathrm{H}$ Snieder

T D Spector

Department of Preventive Ophthalmology,

Institute of

Ophthalmology,

London

C J Hammond

C E Gilbert

Correspondence to: Dr Christopher Hammond ch@twin-research.ac.uk

Accepted for publication 27 April 2000
In clinical practice there is a wide variation in pupil size after mydriasis. Large pupils make cataract surgery easier and quicker ${ }^{1}$ and allow for more thorough examination of peripheral retina, which may be important in the success of retinal detachment surgery. Some conditions are known to reduce the size of dilated pupils, such as diabetes mellitus and pseudoexfoliation. However, it is not known to what extent genes and environment are important in determining the size of pupils in the population.

Twin studies have been described as the "perfect natural experiment" to decide between the relative importance of genetic and environmental factors. ${ }^{2}$ They are based on comparison of the concordance between identical or monozygotic (MZ) twin pairs and nonidentical or dizygotic (DZ) twins. $\mathrm{MZ}$ twins share the same genes and DZ twins share only half of their genes; any greater similarity between $\mathrm{MZ}$ twins can therefore be attributed to this additional genetic sharing. A classic twin study was performed to examine the heritability of pupil size after mydriasis. Dilated pupil size was measured in a large sample of twins and quantitative genetic modelling used to estimate the relative role of genes and environment. In addition, other factors such as the effect of iris colour, age, and refractive error on pupil size were studied.

\section{Methods}

SUBJECTS

In all, 226 monozygotic (MZ) and 280 dizygotic (DZ) female white twin pairs recruited through the St Thomas's UK adult twin registry were examined. They were ascertained from the general population through national media campaigns in the United Kingdom. ${ }^{3}$ Local ethics committee approval was obtained. As part of a study into age related eye disease, twins between the ages of 49 and 79 were invited to attend an eye examination and informed consent was obtained. Zygosity was determined by standardised questionnaire $^{4}$, and confirmed by DNA short tandem repeat fingerprinting in approximately $40 \%$ of twin pairs where zygosity was uncertain. Of the 506 pairs of twins (2024 eyes), 63 eyes were excluded from analysis of pupil size: 24 eyes were pseudophakic, 11 were ungradable due to previous eye surgery or injury and data from 28 eyes was missing.

\section{MEASUREMENTS}

Pupils were dilated with one drop of tropicamide $1 \%$ followed 45 seconds later by one drop of phenylephrine $10 \%$. Eyes were photographed 50-70 minutes later using a digital retroillumination camera (Marcher Instruments Ltd) and pupil size measured by superimposing a standardised grid over these images.

A reproducibility study was performed on 30 of the twins: retroillumination photographs were repeated 1-6 months after the initial visit. The intraclass correlation between pupil size on the two occasions was $0.89(p<0.001)$. A further repeatability study of the assessment of the images was performed when pupil sizes were measured again from 60 randomly chosen images: the intraclass correlation coefficient was $0.97(p<0.001)$.

ANALYTICAL APPROACH

Details of model fitting to twin data have been described elsewhere. ${ }^{56}$ In short, the technique is based on the comparison of the variance covariance matrices in $\mathrm{MZ}$ and $\mathrm{DZ}$ twin pairs 


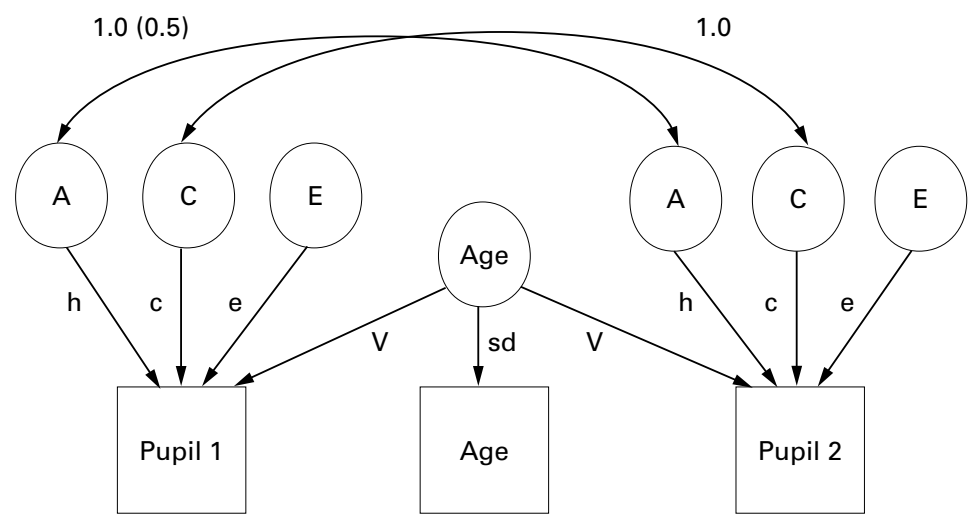

Figure 1 Path model for the measured variables of dilated pupil sizes for the right eye of twin 1 (pupil 1) and twin 2 (pupil 2) and age which are represented in squares. Latent factors are represented in circles: $A, C$, and $E$ are the additive genetic, common environmental, and unique environmental influences. $D$, the dominant genetic influence, was also tested but is omitted to simplify the diagram. The correlation between the latent genetic factors is 1 for $M Z$ pairs and 0.5 for $D Z$ pairs. For the common environmental factors it is 1 for $M Z$ and $D Z$ pairs. Regression coefficients of the observed variables on the different latent factors are shown in lower case: $h$ is the additive genetic effect, $V$ the age effect, $c$ the common environment effect, e the unique environmental path coefficient, and sd the standard deviation of age.

and allows separation of the observed phenotypic variance into additive (A) or dominant (D) genetic components and common (C) or unique (E) environmental components using structural equation modelling. E also contains measurement error. Dividing each of these components by the total variance yields the different standardised components of variance-for example, the heritability $\left(h^{2}\right)$ which can be defined as the ratio of additive genetic variance to total phenotypic variance.

As twins share the same age, correlations for both $M Z$ and DZ pairs will be inflated if age significantly affects pupil size. If not accounted for, the effect of age is confounded with C, their common environment. ${ }^{7}$ As age was correlated with pupil size, it was incorporated into the model to allow estimation of its effect on the variance within the population. Figure 1 illustrates the twin model used for analysis.

MODEL FITTING PROCEDURE

A series of models were fitted to the variance covariance matrices. The significance of variance components A, C, D, and age was assessed by testing the deterioration in model fit after each component was dropped from the full model, leading to a model with as few parameters as possible. Submodels were compared with the full model by hierarchic $\chi^{2}$ tests. The difference in $\chi^{2}$ values between submodel and full model is itself approximately distributed as $\chi^{2}$, with degrees of freedom (df) equal to the difference in df of submodel and full model. Model selection was also guided by Akaike's information criterion (AIC $=\chi^{2}-$ $2 \mathrm{df}$ ). The model with the lowest AIC reflects the best balance between goodness of fit and parsimony.

STATISTICAL SOFTWARE

Data handling and preliminary analyses were done with sTATA. ${ }^{8}$ All genetic modelling was carried out with $\mathrm{Mx} .^{9}$

\section{Results}

The mean age of the $226 \mathrm{MZ}$ twin pairs was 62.4 years (SD 5.7) with a range of 51-75 years and the mean age of the $280 \mathrm{DZ}$ twins was 62.1 years (SD 5.7) ranging from 49 to 79. Table 1 displays the mean (SD) pupil size for the subjects and the number of twin pairs included in each analysis. Pupil sizes were similar for right and left eyes and in $\mathrm{MZ}$ and DZ twin pairs. Scatter plots for right pupil size for $M Z$ and $D Z$ twin pairs (Fig 2) demonstrated a stronger correlation for $\mathrm{MZ}$ twin pairs. The intraclass correlation coefficients for pupil size reflecting within twin similarity confirmed this (Table 1): the $\mathrm{MZ}$ correlation of 0.82 is double the $\mathrm{DZ}$ correlation of 0.39 , suggesting an important genetic effect on pupil size after mydriasis.

Other factors that might affect pupil size were eye colour, age, and refractive error. Table 2 demonstrates that iris colour had no clinically significant effect on pupil size. The overall mean pupil size was $7.80 \mathrm{~mm}$, with blue eyes dilating least, but they dilated only 0.1 $\mathrm{mm}$ less than brown, green, and hazel irides. Pupil size after mydriasis tended to decrease with age, with a correlation coefficient of -0.15 . Age was therefore included in subsequent genetic modelling. Pupil size was weakly and inversely correlated with spherical equivalent $(r=-0.10)$, but modelling showed that refractive error contributed less than $1 \%$ to the variance of pupil size, so this was excluded from further analysis.

Univariate modelling of pupil sizes was performed (Table 3). The best fitting AE model suggested that the variance of pupil size is explained by additive genes and unique environment. Both C and D (common family environment and dominant genetic effect) could be dropped with no significant loss of fit, but if the effect of A (additive genes) or age was removed there was a significant loss of fit.

Standardised parameter estimates for the best fitting models are listed in Table 4. It shows the heritability of dilated pupil size was $78-80 \%$. Age only explained $2-3 \%$ of the variance, with the rest of the variance (18-19\%) explained by unique environment.

\section{Discussion}

Pupil size after mydriasis is strongly heritable, with additive genes explaining up to $80 \%$ of the

Table 1 Mean and range of pupil sizes for twin pairs after exclusions

\begin{tabular}{|c|c|c|c|c|c|c|c|c|}
\hline \multirow[b]{2}{*}{ Eye } & \multicolumn{4}{|c|}{$M Z$ twin pairs } & \multicolumn{4}{|c|}{$D Z$ twin pairs } \\
\hline & $N$ & Mean (SD) & Range & $I C C$ & $N$ & Mean (SD) & Range & ICC \\
\hline Right & 213 & $7.79(0.73)$ & $4.7-9.5$ & 0.82 & 270 & $7.79(0.69)$ & $5.2-9.5$ & 0.39 \\
\hline Left & 216 & $7.82(0.70)$ & $4.6-9.5$ & 0.82 & 268 & $7.81(0.70)$ & $4.2-9.5$ & 0.39 \\
\hline
\end{tabular}

$\mathrm{MZ}=$ monozygotic, $\mathrm{DZ}=$ dizygotic $\mathrm{N}=$ number of twin pairs; $\mathrm{ICC}=$ intraclass correlation coefficient within twin pairs. 
MZ twin pairs

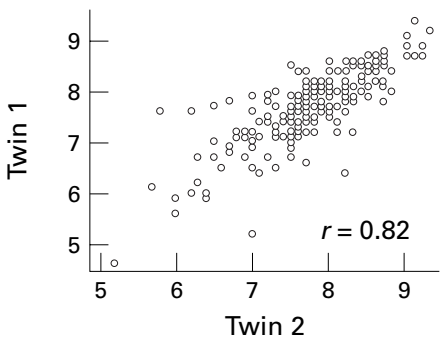

DZ twin pairs

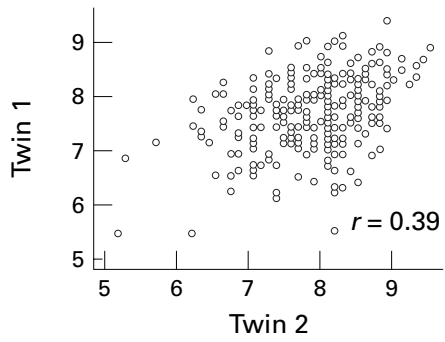

Figure 2 Scatter plots of the pupil sizes of the right eye plotted for twin 1 against twin 2 in $M Z$ twin pairs (left) and DZ twin pairs (right). $r=$ correlation coefficient.

variance of pupil size in this population. Unique environment, which includes factors such as variation in dose of drug administered, measurement error, and an individual's unique environmental circumstances, explains 18 $19 \%$. Age is only responsible for $2-3 \%$ of the variance of pupil size.

The high correlation within $M Z$ twin pairs in this study is similar to the only previous twin study of five $\mathrm{MZ}$ and five $\mathrm{DZ}$ twin pairs which showed a correlation for non-dilated pupil size as well as the effect of phenylephrine. ${ }^{10}$ That study showed no significant correlation for DZ twins, which is surprising for an inherited trait since DZ twins share $50 \%$ of their genetic material as $\mathrm{MZ}$ twins, but undoubtedly reflects the insignificant numbers.

Pupil size was measured at around 60 minutes after instillation of the mydriatics, the time that has been reported for maximal effect of phenylephrine alone ${ }^{11}$ or in combination with tropicamide. ${ }^{12}$ The high reproducibility

Table 2 Pupil sizes for different eye colours

\begin{tabular}{|c|c|c|c|}
\hline & & Right eye & Left eye \\
\hline Colour & No (\%) & Mean (SD) & Mean (SD) \\
\hline Blue & $457(46)$ & $7.72(0.73)$ & $7.76(0.71)$ \\
\hline Brown & $203(20)$ & $7.85(0.68)$ & $7.88(0.69)$ \\
\hline Green & $147(15)$ & $7.83(0.65)$ & $7.83(0.64)$ \\
\hline Hazel & $190(19)$ & $7.87(0.71)$ & $7.86(0.72)$ \\
\hline
\end{tabular}

No $=$ numbers of eyes in each group.

Table 3 Univariate modelling of pupil size for right and left eyes

\begin{tabular}{llrllr}
\hline Eye & Model & \multicolumn{1}{c}{$\chi^{2}$} & p Value & $d f$ & \multicolumn{1}{c}{ AIC } \\
\hline Right & ACE and age & 5.207 & 0.635 & 7 & -8.793 \\
& ACE no age & 22.694 & 0.004 & 8 & 6.694 \\
& ADE and age & 5.166 & 0.640 & 7 & -8.834 \\
& AE and age & 5.207 & 0.735 & 8 & -10.793 \\
& CE and age & 80.261 & 0 & 8 & 64.261 \\
Left & & & & \\
& ACE and age & 4.751 & 0.690 & 7 & -9.249 \\
& ACE no age & 17.773 & 0.023 & 8 & 1.773 \\
& ADE and age & 4.522 & 0.718 & 7 & -9.478 \\
& AE and age & 4.751 & 0.784 & 8 & -11.249 \\
& CE and age & 86.968 & 0 & 8 & 70.968 \\
\hline
\end{tabular}

$\chi^{2}=\chi^{2}$ goodness of fit statistic; $\mathrm{df}=$ degrees of freedom, $\mathrm{p}=$ probability; AIC $=$ Akaike's information criterion. See text for further abbreviations.

Table 4 Standardised parameter estimates and 95\% confidence intervals of the best fitting AE model of univariate analysis of pupil size for right and left eyes

\begin{tabular}{lllllll}
\hline Eye & $h^{2}$ & $95 \% C I$ & $e^{2}$ & $95 \% C I$ & Age & $95 \% C I$ \\
\hline Right & 0.78 & $0.73-0.82$ & 0.19 & $0.15-0.23$ & 0.03 & $0.008-0.06$ \\
Left & 0.80 & $0.75-0.84$ & 0.18 & $0.15-0.23$ & 0.02 & $0.004-0.05$ \\
\hline
\end{tabular}

$\mathrm{h}^{2}=$ proportion of variance due to additive genes, $\mathrm{e}^{2}=$ proportion of variance due to individual environmental effects, age $=$ proportion of variance due to age effect, $95 \% \mathrm{CI}=95 \%$ confidence interval.

score in this study agrees with other studies of the reproducibility of pupil size after mydriasis within individuals. ${ }^{13}$ This study only examined final pupil size, and so it cannot be certain whether the strong genetic influence is on actual maximum pupil size or on the response to mydriatics. Bertler and Smith's previous twin study found a high $\mathrm{MZ}$ correlation for initial pupil size and no difference in the rate of dilatation between $\mathrm{MZ}$ and $\mathrm{DZ}$ twins, ${ }^{10}$ suggesting that the genes determine mydriasis not in response to drug or dose, but determine the maximal possible size of the pupil. If pupil size is largely genetically determined, the tendency among surgeons to instil further mydriatics when a pupil does not dilate well, with potential for systemic toxicity, may be inappropriate. However, this study did not assess whether further doses would influence final pupil size.

Although the $\mathrm{MZ}$ twins are highly correlated they are not exactly the same. Even in traits that are highly heritable, such as height, the correlation is rarely above $0.8-0.9$, and for greater similarity one would have to conjecture a $100 \%$ heritable trait with $100 \%$ penetrance with no measurement error effects or environmental influence. Classic twin studies estimate the contribution of genes and environment to a trait's variance in a population, but cannot separate measurement error, dose effects, and other possible environmental effects in individuals.

The observation that dilated pupil size decreases with age and that it does not correlate with iris colour confirms other studies. ${ }^{12}$ Although myopic eyes are significantly longer than hypermetropic eyes, refractive error contributed less than $1 \%$ to the variance of dilated pupil size, suggesting that the scleral enlargement of myopia does not significantly affect final pupil size. Pseudophakic individuals were excluded from this study, as cataract surgery has been shown to affect pupil size, ${ }^{14}$ although with modern phacoemulsification procedures causing less iris trauma than previous surgical techniques, this may be less relevant.

This twin study should be generalisable to the population as twins on the whole do not differ from singletons. ${ }^{15}$ However, heritability is a population specific estimate: our figure applies to this population of middle aged British white women and may differ for other ethnic groups ${ }^{16}$ or those with different environmental circumstances.

In conclusion, pupil size after mydriasis is strongly genetically determined, and this explains the wide variation seen in clinical practice. Age explains up to 3\% of the variance, but iris colour and refractive error have little effect on dilated pupil size. Whether different doses of mydriatics are needed in genetically diverse individuals remains to be seen.

We would like to acknowledge the twin volunteers who participated in this study, which was funded by the Wellcome Trust. The St Thomas' UK Adult Twin Registry also receives support from the Arthritis Research Campaign, British Heart Foundafrom the Arthritis Research Campaign, British Heart Founda-
tion, Chronic Disease Research Foundation, and Gemini tion, Chronic Disease Research Foundation, and Gemini
Research Ltd. We would like to thank Pauline Rook and Carol Research Ltd. We would like to th
Deller for administrative support. 
1 Joseph J, Wang HS. Phacoemulsification with poorly dilated pupils. F Cataract Refract Surg 1993;19:551-6.

artin N, Boomsma D, Machin G. A twin-pronged attack on complex traits. Nat Genet 1997;17:387-92.

Boomsma DI Twin registers in Europe: an overview. Twin Research 1998;1:34-51.

4 Martin NG, Martin PG. The inheritance of scholastic abilities in a sample of twins. I. Ascertainments of the sample and diagnosis of zygosity. Ann Human Genet 1975;39:21318.

5 Neale MC, Cardon LR. Methodology for genetic studies of twin and families. Dordrecht: Kluwer Academic Publishers, 1992.

6 Snieder H, Boomsma DI, van Doornen LJP. Heritability of respiratory sinus arrhythmia: dependency on task and respiration rate. Psychophysiology 1997;34:317-28.

7 Snieder $\mathrm{H}$. Path analysis of age-related disease traits. In: Spectin Media, 1999:119-30.

8 StataCorp. Intercooled Stata for Windows 95. (5.0). 1997. College Station, StataCorp.
9 Neale MC. Mx:statistical modeling. 4th ed. Box $126 \mathrm{MCV}$, Richmond, VA: Dept of Psychiatry, Medical College of Richmond, VA:

10 Bertler A, Smith SE. Genetic influences in drug responses of the eye and the heart. Clin Sci 1971;40:403-10.

11 Levine L. Effective degree of mydriasis with phenylephrine and tropicamide. Am f Optom Physiol Opt 1976;53:774-85.

12 Apt L, Henrick A. Pupillary dilatation with single eyedrop mydriatic combinations. Am $\mathcal{F}$ Ophthalmol 1980;89:553-9.

13 Maclean H. Reproducibility of mydriasis. Ophthalmic Res 1992;24(Suppl 1):36-9.

14 Gibbens MV, Goel R, Smith SE. Effect of cataract extraction on the pupil response to mydriatics. $\mathrm{Br} F$ Ophthalmol 1989;73:563-5.

15 Kyvik KO. Generalisability and assumptions of twin studies. In: Spector TD, Snieder H, MacGregor AJ, eds. Advances in twin and sib-pair analysis. London: Greenwich Medical Media, 1999:67-77.

16 Emiru VP. Response to mydriatics in the African. $\mathrm{Br} \mathcal{F} \mathrm{Oph}$ thalmol 1971;55:538-43. 